\title{
Références bibliographiques du dossier « enseigner la diversité culturelle»
}

\section{Marie-Geneviève Picart}

\section{Q OpenEdition}

1 Journals

Édition électronique

URL : http://journals.openedition.org/ries/2965

DOI : 10.4000/ries.2965

ISSN : 2261-4265

Éditeur

Centre international d'études pédagogiques

\section{Édition imprimée}

Date de publication : 1 mars 1998

ISBN : 9771254459005

ISSN : 1254-4590

\section{Référence électronique}

Marie-Geneviève Picart, « Références bibliographiques du dossier « enseigner la diversité culturelle » », Revue internationale d'éducation de Sèvres [En ligne], 17| 1998, mis en ligne le 19 avril 2013, consulté le 22 avril 2019. URL : http://journals.openedition.org/ries/2965 ; DOI : 10.4000/ries.2965

Ce document a été généré automatiquement le 22 avril 2019

(c) Tous droits réservés 


\title{
Références bibliographiques du dossier " enseigner la diversité culturelle»
}

\author{
Marie-Geneviève Picart
}

1 Le thème de la diversité culturelle étant au croisement de nombreux domaines de recherche, nous avons renoncé au classement thématique, peu pertinent dans ce cadre. Nous avons privilégié les ouvrages et articles récents et faisant le point sur l'état actuel de la recherche ainsi que sur quelques titres fondamentaux indispensables à la compréhension de la problématique. Les références bibliographiques comportant un résumé sont consultables au CIEP.

2 ABDALLAH-PRETCEILLE Martine, PORCHER Louis, Éducation et communication interculturelle, Paris, PUF, «L'éducateur », 1996, 192 p., bibliogr.

La variété des publics au sein de l'institution éducative, les appartenances sociales et historiques multiples, la coexistence d'identités différentes caractérisent les systèmes éducatifs d'aujourd'hui. La première partie de l'ouvrage examine ces mutations pour rechercher une cohésion éducative. La seconde partie précise « les termes dans lesquels se pose l'altérité quand elle est conjuguée avec la diversité culturelle ».

3 ABDALLAH-PRETCEILLE Martine (dir.), THOMAS Alexander (dir.), Relations et apprentissages interculturels, Paris, A. Colin, "Bibliothèque européenne des sciences de l'éducation», 1995, 178 p., bibliogr.

Actes du colloque sur l'interculturel qui s'est tenu à Amiens en octobre 1990. Les exposés théoriques sur l'identité culturelle, les conflits entre cultures, les relations et les échanges interculturels, sont suivis d'un programme d'activités interculturelles, d'une réflexion sur la compréhension d'une culture étrangère et sur l'identité philosophique européenne.

4 ABOU Sélim, L'identité culturelle : relations interethniques et problèmes d'acculturation, Paris, Hachette, « Pluriel », 1995, 249 p. (éd. de 1981 chez Anthropos).

$\mathrm{Au}$ sommaire de l'ouvrage, les relations interethniques, les principes de politique ethnolinguistique, les idéologies et les mythes de l'acculturation. 

sur la formation aux échanges scolaires/Strasbourg, La pédagogie des échanges. Buts et moyens de la formation des enseignants : se rencontrer pour se former, se former à se rencontrer, Strasbourg, Conseil de l'Europe, 1993, 65 p.

La pédagogie des échanges, devenue pédagogie de la rencontre, a créé un espace pédagogique nouveau et pluri référentiel. Pédagogie de la complexité, elle suppose l'invention d'outils didactiques visant l'acquisition d'une compétence communicative et la volonté de partage d'une culture. Cette publication rend compte du travail réalisé par le GREFES sur les plans conceptuel et expérimental.

6 Au cœur des cultures, Sciences humaines, 1997/11, $n^{\circ}$ 77, p. 18-35, ill, bibliogr.

Le mot culture désigne-t-il, comme le veut l'usage courant, un héritage ancien, fondateur des peuples et de leurs traditions? Fait-il référence à la propriété d'un groupe ? Ou désigne-t-il nécessairement ce.qui différencie le plus les hommes entre eux? Autant de questions traitées par les quatre contributions de ce dossier.

BANKS James A. (ed.), Multicultural Education, Transformative Knowledge, and Action: Historical and Contemporary Perspectives, New York, Teachers College Press, 1996, 372 p.

BYRAM Michael, Culture et éducation en langue étrangère, Paris, Didier, « Langues et apprentissage des langues » (LAL), 1992, 220 p., index, bibliogr.

L'acquisition d'une compétence interculturelle s'inscrit dans une finalité pratique et doit constituer une part essentielle dans l'éducation générale d'un individu. Cet ouvrage propose un modèle intégré d'enseignement des langues et des cultures.

9 BYRAM Michael, ZARATE Geneviève, NEUNER Gerhard, La compétence socioculturelle dans l'apprentissage et l'enseignement des langues, Strasbourg, Conseil de l'Europe, 1997, 124 p.

En deux études, les auteurs examinent la manière dont la compétence socioculturelle peut être décrite et évaluée, afin de permettre aux apprenants en langues de rendre pleinement compte de leur potentiel pour la communication interculturelle, essentielle pour une citoyenneté européenne.

10 CAMILLERI Carmel, "À quelles conditions peut-on parler d'un culture européenne?", Intercultures, 1994, $n^{\circ}$ 25-26, p. 7-27.

Considérant d'abord ce qu'il appelle culture du premier degré, l'auteur postule qu'il est impossible d'exhumer d'une mémoire collective, pour de quelconques " retrouvailles », une culture européenne préexistante. À un second degré, celui des nations, la durabilité d'une culture commune ne pourrait se fonder que sur un modèle étendu des valeurs de chacune des composantes et, plutôt que de vouloir perpétuer le passé, devra se reposer sur une « utopie enracinée » (Paul Ricœur). Le développement des contacts de porteurs de ces cultures rendra, de fait, essentielle une politique européenne des relations interculturelles.

11 CAMILLERI Carmel, Différence et cultures en Europe, Strasbourg, Conseil de l'Europe, 1995, $221 \mathrm{p}$.

Le document a été rédigé dans le cadre du projet «Démocratie, droits de l'homme et minorités : aspects éducatifs et culturels ». Il se compose de trois parties : l'altérité et les différences culturelles, les sociétés pluriculturelles et l'interculturalité, le multiculturalisme et les institutions. 
12 CAMILLERI Carmel, "La culture et l'identité culturelle: champ notionnel et devenir", in: CAMILLERI Carmel (dir.), COHEN-EMERIQUE Margalit (dir.) : Chocs de cultures : concepts et enjeux pratiques de l'interculturel, Paris, L'Harmattan, 1989, p. 21-73.

Exposé sur des notions théoriques fondamentales : la culture et les concepts annexes, le changement culturel et ses conséquences sur le social et l'individuel, les problèmes identitaires.

CENTRE RÉGIONAL DE DOCUMENTATION PÉDAGOGIQUE, Enseigner l'histoire des religions dans une démarche laïque : représentations, perspectives, organisation des apprentissages : actes du colloque international de Besançon, 20-21 novembre 1991, Besançon, CRDP, Documents, actes et rapports pour l'éducation, 1992, 347 .

CEREZAL Fernando, "Modem Languages and Interculturality in the Primary Sector in England, Greece, Italy and Spain ", Mediterranean Journal of Educational Studies, 1997, vol 2, n² 2, p. 1-16, bibliogr.

Cet article présente divers aspects de l'apprentissage des langues modernes dans l'enseignement primaire de trois pays méditerranéens (Grèce, Italie, Espagne) et dans celui de l'Angleterre. Les réformes éducatives en cours concernant l'apprentissage des langues et l'interculturalité sont prises en compte.

COLIN Lucette (dir.), MULLER Burkhard, La pédagogie des rencontres interculturelles, Paris, Anthropos, Office franco-allemand pour la jeunesse, 1996, $297 \mathrm{p}$.

Les contributions rassemblées dans l'ouvrage relatent des expériences vécues dans le cadre d'échanges franco-allemands et plus largement dans celui des échanges internationaux de jeunes. La première partie de l'ouvrage est centrée sur l'apprentissage de la différence. Les contributions de la deuxième partie analysent les supports pédagogiques pouvant assurer une médiation avec la culture étrangère: échanges scolaires, apprentissage précoce des langues étrangères, contenu des manuels scolaires, rôle de la traduction dans la communication interculturelle. La troisième partie est centrée sur les pratiques et les formes de la pédagogie des rencontres internationales. La dernière partie traite les perspectives et les enjeux d'une véritable pédagogie des apprentissages interculturels.

COLLOT Andrée (dir.), DIDIER Guy (dir.), LOUESLATI Béchir (dir.), La pluralité culturelle dans les systèmes éducatifs européens, Nancy, CRDP, 1993, 326 p.

Les nombreuses réflexions et expériences présentées ici sont celles du colloque international tenu à Nancy en janvier 1992 sur le thème « La pluralité culturelle dans les systèmes éducatifs européens ». Cet état des lieux de l'interculturalité dans les systèmes d'enseignement met en évidence les traits communs et les spécificités.

17 COMMUNAUTÉS EUROPÉENNES/COMITÉ DES RÉGIONS, Avis du Comité des régions sur "l'éducation interculturelle ", Luxembourg, Journal officiel des Communautés européennes, 1997/07, 7 p.

Dans le cadre de "l'Année européenne contre le racisme ", le Comité des régions donne son avis sur les types de réponse que doivent apporter les systèmes éducatifs aux problèmes du racisme et de la xénophobie, dans une Europe pluraliste et multiculturelle.

18 COSTE Daniel (coord.), "Éduquer pour une Europe des langues et des cultures?", Études de linguistique appliquée, 1997/06, $n^{\circ}$ 106, p. 133-246, bibliogr.

Les articles regroupés dans ce numéro traitent des questions en cours autour de l'éducation à l'Europe, de la communication interculturelle en milieu scolaire européen, 
de l'éducation à une citoyenneté européenne, de la dimension européenne et des compétences culturelles dans l'enseignement des langues. Sur ce dernier sujet, un rapide cadre de référence est proposé sur le modèle de celui des compétences langagières. L'un des articles défend la thèse selon laquelle la bipolarisation enseignement précoce/ enseignement bilingue consacrerait un enseignement à deux vitesses.

DADSI Driss, Particularisme et universalisme : la problématique des identités, Strasbourg, Conseil de l'Europe, 1995, $129 \mathrm{p}$.

Comment une société peut-elle maintenir sa cohérence nationale et/ou régionale, tout en respectant la diversité des minorités installées sur son territoire? Comment les minorités peuvent-elles maintenir, voire développer une identité autonome, tout en s'intégrant, pour ce qui est de l'espace politico-juridique, à la société globale ?

DE CARLO Maddalena, L'interculturel, Paris, Clé international, "Didactique des langues étrangères ", 1998, 126 p., bibliogr.

L'ouvrage fait le point sur certains concepts très utilisés en didactique des langues (culture, authenticité, stéréotype, identité) et suggère des pistes de travail en classe, dans une optique interculturelle. Définition critique du stéréotype et concept d'identité narrative sont exposés.

21 DEMORGON Jacques, Complexité des cultures et de l'interculturel, Paris, Anthropos, "Exploration interculturelle et science sociale ", Office franco-allemand pour la jeunesse, 1996, $318 p$.

S'appuyant sur son expérience, l'auteur expose ici ce que sont les «nouvelles approches de la culture »: approche synchronique et logique des antagonismes, approche diachronique, approche stratégique et organisationnelle, approches "dimensionnelles » et «sectorielles ». Puis il tente de définir ce qu'il appelle une génétique rétrospective des cultures, sur les exemples allemand et français. Le dernier chapitre, intitulé « Cultures et mondialisations", étudie des concepts tels que «national-marchand», « informationnel », « interculturel mondial », « génétique interculturelle ».

DONDELINGER Germain (éd.), WENGLER André (éd.), Plurilinguisme et identité culturelle : actes des assises européennes pour une éducation plurilingue, Luxembourg, 3-6 novembre 1993, Louvain-la-Neuve, Peeters, "Bibliothèque des Cahiers de l'Institut de linguistique de Louvain ", 1995, $182 \mathrm{p}$.

DRAGONAS Thalia, FRANGOUDAKI Anna, INGLESSI Chryssi, Beyond One's Own

Backyard : Intercultural Teacher Education in Europe (De chez moi et d'ailleurs: éducation interculturelle des enseignants en Europe), Athènes, Society for the Study of Human Sciences, 1996, 246 p.

Échange d'expériences sur la formation des enseignants et les pratiques interculturelles en éducation dans les pays de l'Union européenne.

GAUDET Edithe, LAFORTUNE Louise, Pour une pédagogie interculturelle: des stratégies d'enseignement, St-Laurent (Canada), Erpi, 1997, 426 p.

L'ouvrage se focalise sur le thème de l'enseignement de la diversité culturelle à partir des expériences scolaires menées au Québec: sensibilisation aux différentes conceptions du temps, de l'espace, et aux normes de comportement. Les nombreuses fiches pédagogiques décrivent le travail élaboré en ateliers.

GIRODET Marie-Alix, L'influence des cultures sur les pratiques quotidiennes de calcul, Paris, Didier, «Essais », 1996, $161 p$. 
L'auteur étudie les variations culturelles dans le cadre de l'enseignement des mathématiques. Les systèmes de numération parlée, de mesures et de calculs écrits ou mentaux diffèrent selon les pays dans lesquels ils sont enseignés.

GRANT Nigel, Some Problems of Identity and Education: a Comparative Examination of Multicultural Education, Comparative Education, 1997/03, vol. 33, n 1, p. 9-28.

GROUX Dominique (coord.), SCHRIEWER Jürgen (coord.), L'éducation comparée, Revue française de pédagogie, 1997/12, n 121, 198 p., bibliogr.

Ce dossier réunit des articles sur l'éducation comparée (perspective historique, approches actuelles, perspectives de développement) et des études comparatives entre différents pays (systèmes éducatifs français et japonais, éducation et croissance économique en France et en Allemagne, éducation à la citoyenneté au Canada, aux États-Unis et en France).

HONOR Monique, Enseigner et apprendre dans une classe multiculturelle: méthodes et pratiques pour réussir, Lyon, Chronique sociale, "L'essentiel. Pédagogie formation ", 1996, 171 p.

Gestion des problèmes relationnels, d'une part, pédagogiques, d'autre part, sont les deux grandes questions posées dans ce type de classe. L'auteur propose une démarche d'analyse des problèmes qui part de la pratique et fonctionne à la fois en termes de système et d'interculturel.

HUNTINGTON Samuel, Le choc des civilisations (Trad. de "The clash of civilizations", 1996), Paris, Odile Jacob, 1997, $402 p$.

La politique internationale, sous l'effet de la modernisation, est en train de se redéfinir sur des bases culturelles. Les frontières politiques se redessinent de plus en plus pour rejoindre les frontières ethniques, religieuses et civilisationnelles. Les conflits actuels se produisent « le long des lignes de faille entre civilisations».

KODRON Christoph (éd.), OOMEN-WELKE Ingelore (éd.), Enseigner l'Europe dans nos sociétés multiculturelles (Teaching Europe in Multicultural Society), Freiburg-im-Breisgau (Allemagne), Filibach Verlag, 1995, 451 p., bibliogr.

Plusieurs articles dans cet ouvrage multilingue: l'école, les régions, la migration, les préjugés et les langues. D'autres contributions analysent l'organisation de la concertation européenne, l'introduction d'une dimension internationale dans la formation des enseignants. Une bibliographie commentée, venant de cinq pays, conclut l'ensemble.

KRUMM Hans-Jürgen (coord.), Formation des enseignants à l'éducation multilingue et multiculturelle (Teacher Training for Multilingual and Multicultural Education/Lehreraus- und Fortbildung für eine Mehrsprachige und multikulturelle Erziehung), Workshop n², 24-29 April 1995, Graz (Autriche), Centre européen pour les langues vivantes du Conseil de l'Europe, 1995/06, 10 p. + annexes.

Le Centre européen pour les langues vivantes de Graz est une institution du Conseil de l'Europe dont l'objectif est de promouvoir le plurilinguisme en Europe. Des conférences, des colloques et séminaires favorisent l'échange d'informations et la coopération dans le domaine de la politique des langues.

LANDERCY A. (éd)., RENARD Raymond (éd.), Aménagement linguistique et pédagogie interculturelle, Paris, Didier Érudition, Mons, Centre international de phonétique appliquée, 1996, 256 p. bibliogr.

Les travaux réunis ici sont orientés essentiellement vers l'Afrique. La problématique de l'aménagement linguistique dans des situations éducatives plurilingues est exposée dans 
toute sa complexité: statut des langues minoritaires, articulation des langues de scolarisation, sensibilisation et formation des enseignants, production de matériel pédagogique adéquat. L'enseignement plurilingue peut jouer un rôle essentiel dans la compréhension de la diversité culturelle.

LE THANH KHOI, Éducation et civilisations : sociétés d'hier, Paris, Nathan, 1995, 704 p., bibliogr.

Cet ouvrage retrace l'histoire des relations entre l'éducation et la société dans les différentes civilisations du passé. Il aborde successivement l'histoire de l'éducation dans les sociétés communautaires d'Afrique, au sein des cultures hindoue et bouddhique d'Asie, dans la société mandarinale et féodale de Chine et du Japon, en Europe, de la Grèce antique à la chrétienté médiévale, dans le monde islamique, à la fois un et divers. Ce panorama constitue un apport important aux études historiques en matière d'éducation comparée.

PEMBROKE Emmanuelle de, À la découverte d'une culture étrangère: de la connaissance à la compétence relationnelle, Babylonia, 1997, n³ 3, p. 84-88, bibliogr.

L'auteur étudie le rapport qu'entretiennent langue et culture et l'importance d'enseigner conjointement ces deux dimensions. Elle propose également une démarche favorisant l'approche et la compréhension d'une culture étrangère.

PEROTTI Antonio, Plaidoyer pour l'interculturel, Strasbourg, Conseil de l'Europe, 1994, 150 p., bibliogr.

Cet ouvrage a pour objectif de présenter une synthèse des réflexions menées dans les travaux du Conseil de l'Europe en matière d'éducation interculturelle. Il fait d'abord un état des lieux sur les sociétés européennes (nationalismes, diasporas, identités culturelles, immigration en Europe), redéfinit quelques notions de base, donne des jalons pour une éducation interculturelle à l'école (en général et par disciplines) et élargit ensuite la réflexion en vue de la définition d'une politique de l'éducation.

PHILIPP Marie-Gabrielle (coord.), PREVOST Patrick (coord.), GIRAUD Michel (coord.), De l'approche interculturelle en éducation, Éducation et pédagogie, 1993/09, n 19, 102 p., bibliogr.

Ces actes de l'université d'été tenue en juillet 1992 sur le thème "Quelle éducation interculturelle pour quelle intégration? » regroupent diverses contributions (Conseil de l'Europe, CIEMI, ministère des Affaires sociales, FAS, IREMAM, etc.) portant sur les perspectives européennes (comparaison entre la France et le Royaume-Uni) et la politique française en matière d'éducation interculturelle. La dernière partie offre un éclairage notionnel (pluralisme, droits de l'homme, intégration). La bibliographie regroupe les textes officiels français (rapports et synthèses) et ceux du Conseil de l'Europe sur le sujet.

PORCHER Louis (coord.), Cultures, culture..., Le Français dans le monde: recherches et applications, 1996/01, $160 \mathrm{p}$.

Nous assistons depuis quelques années dans le cadre de l'enseignement des langues, à une montée forte des enjeux culturels jusqu'ici quelque peu délaissés. Ce numéro a sélectionné de façon subjective, cinq angles de vues sur les pratiques culturelles: les " cultures cultivées », les « cultures médiatiques », les «cultures anthropologiques », les «cultures managériales» et les "cultures invisibles». Pour ces cinq orientations, un équilibre a été souhaité entre contributions formelles et entretiens.

RASSEKH Shapour, Éducation et culture de la paix: sélection bibliographique mondiale, Unesco, Bureau international d'éducation, 1996, $317 \mathrm{p}$. 
World Trends in Policy, Law, and Administration, Berkeley, Institute of Governmental Studies Press, Berlin, Max Plank institute for Human Development and Education, 1995, $345 \mathrm{p}$.

de formation, Apprentissage des langues et citoyenneté européenne et Campagne européenne de la jeunesse contre le racisme, la xénophobie, l'antisémitisme et l'intolérance, Strasbourg, Conseil de l'Europe, 1996, 66 p.

Les enseignants de langues ont un rôle central à jouer dans la préparation des jeunes à une citoyenneté active. Les auteurs présentent ici des thèmes et des activités pratiques pour étudier les dimensions linguistiques et culturelles de l'altérité : comportements envers les langues, situation de gêne ou de malentendu, reconnaissance des stéréotypes, symboles de l'identité nationale, frontières, différences culturelles.

CENTRE EUROPÉEN DE LA JEUNESSE, Kit pédagogique: idées, ressources, méthodes et activités pour l'éducation interculturelle informelle avec des adultes et des jeunes, Strasbourg, Conseil de l'Europe, 1995, 246 p. 
L'ouvrage recense des matériels pratiques et théoriques à l'usage des animateurs, des formateurs, des travailleurs de jeunesse et des enseignants: méthodes, éventail d'activités basées essentiellement sur le travail en groupes et la participation pour l'introduction d'une éducation interculturelle.

CENTRE POUR L'ÉDUCATION INTERCULTURELLE, Outils pédagogiques interculturels: un inventaire sélectif de bons exemples dans l'Union européenne, Gand, Steunpunt Intercultureel Onderwijs, 1997, $176 \mathrm{p}$.

Guide européen du matériel éducatif destiné à l'éducation interculturelle. Cet inventaire a été réalisé dans le cadre du projet " Production d'une banque de données européenne de matériels éducatifs interculturels " lancé en 1994 par l'Union européenne. 148 outils pédagogiques destinés à l'enseignement pré-élémentaire, primaire et secondaire ont été sélectionnés sur la base de la présence d'objectifs interculturels et sur leur qualité didactique.

COWEN John E., English Teacher's Portfolio of Multicultural Activities: Ready-to-Use Lessons and Cooperative Activities for Grades 7-12, New York, Center for Applied Research in Education, $1996,317 p$.

Pour un complément d'informations, on pourra consulter le site web des Éditions du Conseil de l'Europe: http://book.coe.fr/ ainsi que les bases de données de l'Unesco accessibles à partir du site $: \underline{h t t p: / / u n e s c o . o r g}$ 